\title{
Commentary \\ BIS and spectral entropy monitoring during sedation with midazolam/remifentanil and dexmedetomidine/remifentanil
}

Anthony R Absalom and David K Menon

University Division of Anaesthesia, Addenbrookes Hospital, Cambridge CB2 2QQ, UK

Corresponding author: Anthony R Absalom, ara30@wbic.cam.ac.uk

Published: 28 April 2009

Critical Care 2009, 13:137 (doi:10.1186/cc7776)

This article is online at http://ccforum.com/content/13/2/137

(c) 2009 BioMed Central Ltd

See related research by Haenggi et al., http://ccforum.com/content/13/1/R20

\begin{abstract}
Haenggi and colleagues report considerable intra- and interindividual variability in derived electroencephalogram (EEG) parameters (Bispectral Index (BIS), response entropy and state entropy) recorded in volunteers sedated with midazolam or dexmedetomidine infusions titrated to modified Ramsay scores of 2,3 and 4, and a remifentanil infusion at a fixed target concentration. Possible explanations for the low, variable and fluctuating EEG parameters are that volunteers were intermittently asleep, and that remifentanil gave rise to a low amplitude, slowed EEG pattern despite maintained consciousness. BIS and entropy values should be interpreted in combination with clinical findings in patients sedated with these agents.
\end{abstract}

In the previous issue of Critical Care, Haenggi and colleagues [1] report the results of an analysis of Bispectral Index (BIS), response entropy (RE) and state entropy (SE) values recorded in volunteers sedated with midazolam or dexmedetomidine infusions titrated to modified Ramsay scores of 2, 3 and 4, and a remifentanil infusion at a fixed target concentration.

Individualised titration of sedation on the intensive care unit has many benefits, but requires a reliable, objective method of assessing sedation level. Although clinical sedation scales are commonly used, and may improve outcomes [2], at best they offer an intermittent, subjective assessment. In comparison, automated systems using analyses of the electroencephalogram (EEG) potentially provide continuous, objective assessments. To be clinically useful, outputs from such monitors should correlate well with a gold standard measure of sedation, with minimal inter- and intra-individual variability at any level of sedation. Haenggi and colleagues [1] found that while clinical sedation assessments, and plasma and effect-site sedative concentrations were stable, there was considerable variability in the BIS, RE and SE. This discordance requires explanation.

One critical issue is whether cognitive status and sedation level were comparable between volunteers, and stable during each study phase in individual volunteers. Sedation status was formally assessed at the start and end of each EEG recording period. This is reasonable, as clinical assessments suffer from the 'observer effect'. However, Occam's razor suggests that cognitive status was neither stable in individuals, nor completely comparable between individuals, since to accept the opposite demands many more assumptions. Further, the Ramsay scale was designed for use in a different population (critically ill patients), contains terms that are open to interpretation and may have poor inter-rater reliability [3]. As the authors point out, environmental noise and auditory stimuli were likely to cause arousal, particularly in volunteers receiving dexmedetomidine (which produces rousable sedation) [4].

How, then, can we explain the marked variability in BIS and entropy readings, which in some cases rapidly oscillated between values associated with consciousness and values associated with surgical planes of anaesthesia? BIS and entropy monitors were designed to correlate EEG signals with anaesthetists' impressions of depth of sedation and anaesthesia across a wide range of clinical states. Design of these monitors includes the assumption that increasing doses of sedative or hypnotic drugs are associated with a continuum of changes in one or more aspects of cerebral electrical activity (such as amplitude, spectral content or bicoherence $[5,6]$ ) that correlate with depth of sedation and anaesthesia. It is unclear whether these assumptions apply to the trial, and more widely to clinical practice, since loss of

$\mathrm{BIS}=$ Bispectral Index; EEG = electroencephalogram; NREM = non-REM; RE = response entropy; REM = rapid eye movement; SE = state entropy. 
consciousness is an abrupt phenomenon during induction of anaesthesia. Some theoretical models of consciousness are based on the ability of the brain to integrate information [7], as sleep and anaesthesia are associated with loss of this ability $[8,9]$. Such models predict that rapid transitions between consciousness and unconsciousness would result in dramatic fluctuations in synchronisation normally present across regions of the brain [10]. While such a process provides a conceptually appealing basis for the dramatic EEG changes seen, a closer analysis makes this an unlikely explanation. According to the manufacturers, BIS or SE values between 40 and 60 represent surgical planes of anaesthesia. At the deepest level of sedation in the current study, volunteers responded to loud verbal stimuli or shoulder taps, implying only moderate sedation. Despite this, most volunteers had BIS or RE values $<60$, and several had RE values associated with deep anaesthesia $(<40)$.

What possible explanations remain for the low (and often oscillating) values found in this study? As indicated by the authors, sleep is a possible explanation, since transitions between rapid eye movement (REM) and non-REM (NREM) sleep are also associated with such abrupt EEG changes. NREM sleep is characterised by EEG 'sleep spindles' that have low entropy, are morphologically similar to spindles seen during anaesthesia, and result in low BIS and RE values [11]. Within a 20 minute time frame, transitions between a lightly sedated state (associated with beta activation and higher BIS and entropy values) and NREM sleep are plausible, and would result in fluctuations in EEG parameters. Recent evidence suggests that loss and return of consciousness are mediated by different neural pathways, and that activity in orexinergic neurons is associated with return (but not loss) of consciousness [12]. Dexmedetomidine acts on sleep pathways, but does not inhibit activity in orixenergic neurons, which is probably why it causes rousable sedation [13] and may explain some of the variability in EEG parameters seen in this arm of the study. Different mechanisms may underlie low BIS or RE values seen with remifentanil without loss of consciousness. In isolation, remifentanil causes EEG slowing, and, at higher doses, produces a significant increase in power in delta frequencies; both of which will cause lower BIS and RE values [14]. Moreover, low, declining remifentanil concentrations (after complete washout of other agents) have been found to be associated with an active but low amplitude EEG, resulting in the BIS monitor incorrectly classifying the rhythm as burst suppression, causing inappropriately low BIS values [15]. Finally, little is known about the pharmacodynamic interactions among remifentanil, midazolam and dexmedetomidine.

In summary, without corroborating objective evidence (such as functional imaging), it is highly likely that sedation state was not constant during the EEG recordings. Despite this, and more importantly, it is highly unlikely that volunteers were unconscious during the study, and thus inappropriately low
BIS and RE values were recorded in many volunteers. BIS and entropy monitors assess changes in parameters calculated from EEG signals but do not search for patterns or features uniquely associated with any particular level of sedation or anaesthesia. When patients or volunteers are sedated with midazolam and remifentanil or dexmedetomidine and remifentanil combinations, caution is required when interpreting the output of currently available EEG-based monitors. Indeed, the most highly desirable state of intensive care unit sedation - one that mimics natural sleep - may present particular problems to EEG monitors primarily developed to monitor anaesthetic depth. Clearly much work remains to be done before we will have a reliable and objective EEG-based monitor that provides state-specific information about consciousness, sedation and anaesthesia.

\section{Competing interests}

ARA has received an unrestricted travel grant from Aspect Medical Systems Inc.

\section{Acknowledgements}

ARA is supported by the BOC Professorship of the Royal College of Anaesthetists, and the West Anglia Comprehensive Local Research Network. DKM is supported by the BOC Professorship of the Royal College of Anaesthetists, the West Anglia Comprehensive Local Research Network and the Cambridge Biomedical Research Centre.

\section{References}

1. Haenggi M, Ypparila-Wolters H, Hauser K, Caviezel C, Takala J, Korhonen I, Jakob SM: Intra- and inter-individual variation of BIS-index ${ }^{\circledR}$ and Entropy ${ }^{\circledR}$ during controlled sedation with midazolam/remifentanil and dexmedetomidine/remifentanil in healthy volunteers: an interventional study. Crit Care 2009, 13:R20.

2. Brook AD, Ahrens TS, Schaiff R, Prentice D, Sherman G, Shannon W, Kollef MH: Effect of a nursing-implemented sedation protocol on the duration of mechanical ventilation. Crit Care Med 1999, 27:2609-2615.

3. Olson D, Lynn M, Thoyre SM, Graffagnino C: The limited reliability of the ramsay scale. Neurocrit Care 2007, 7:227-231.

4. Venn M, Newman J, Grounds M: A phase II study to evaluate the efficacy of dexmedetomidine for sedation in the medical intensive care unit. Intensive Care Med 2003, 29:201-207.

5. Rampil IJ: A primer for EEG signal processing in anesthesia. Anesthesiology 1998, 89:980-1002.

6. Viertiö-Oja H, Maja V, Särkelä M, Talja P, Tenkanen N, TolvanenLaakso $H$, Paloheimo M, Vakkuri A, Yli-Hankala A, Meriläinen P: Description of the Entropy algorithm as applied in the DatexOhmeda S/5 Entropy Module. Acta Anaesthesiol Scand 2004, 48:154-161.

7. Tononi $\mathrm{G}$ : An information integration theory of consciousness. BMC Neurosci 2004, 5:42.

8. Massimini M, Ferrarelli F, Huber R, Esser SK, Singh H, Tononi G: Breakdown of cortical effective connectivity during sleep. Science 2005, 309:2228-2232.

9. Alkire MT, Hudetz AG, Tononi G: Consciousness and anesthesia. Science 2008, 322:876-880.

10. John ER: A field theory of consciousness. Conscious Cogn 2001, 10:184-213.

11. Sleigh JW, Andrzejowski J, Steyn-Ross A, Steyn-Ross M: The bispectral index: a measure of depth of sleep? Anesth Analg 1999, 88:659-661.

12. Kelz MB, Sun Y, Chen J, Cheng Meng Q, Moore JT, Veasey SC, Dixon S, Thornton M, Funato $\mathrm{H}$, Yanagisawa $M$ : An essential role for orexins in emergence from general anesthesia. Proc Natl Acad Sci USA 2008, 105:1309-1314.

13. Nelson LE, Lu J, Guo T, Saper CB, Franks NP, Maze M: The alpha2-adrenoceptor agonist dexmedetomidine converges on 
an endogenous sleep-promoting pathway to exert its sedative effects. Anesthesiology 2003, 98:428-436.

14. Noh GJ, Kim KM, Jeong YB, Jeong SW, Yoon HS, Jeong SM, Kang SH, Linares O, Kern SE: Electroencephalographic approximate entropy changes in healthy volunteers during remifentanil infusion. Anesthesiology 2006, 104:921-932.

15. Muncaster AR, Sleigh JW, Williams M: Changes in consciousness, conceptual memory, and quantitative electroencephalographical measures during recovery from sevoflurane- and remifentanil-based anesthesia. Anesth Analg 2003, 96:720725 . 